\title{
SMEI direct, 3-D-reconstruction sky maps, and volumetric analyses, and their comparison with SOHO and STEREO observations
}

\author{
B. V. Jackson, P. P. Hick, A. Buffington, M. M. Bisi, and J. M. Clover \\ Center for Astrophysics and Space Sciences, University of California, San Diego, 9500 Gilman Drive \#0424, La Jolla, \\ 92093-0424 CA, USA
}

Received: 15 June 2009 - Revised: 2 October 2009 - Accepted: 23 October 2009 - Published: 2 November 2009

\begin{abstract}
In this paper we present the results of the analysis of the late January 2007 Coronal Mass Ejection (CME) events recorded by the Solar Mass Ejection Imager (SMEI), the Solar TErrestrial RElations Observatory (STEREO), and the SOlar and Heliospheric Observatory (SOHO) spacecraft. This period occurs when the two STEREO spacecraft views are from close to Earth, and thus the views from both SMEI and the STEREO outer Heliospheric Imagers (HI-2s) coincide. Three-dimensional (3-D) analyses derived from SMEI data show many CMEs that have also been studied by others using short-term image subtractions (image-differencing techniques). During this interval we map several CME structures that are observed in both SMEI and the STEREO-A HI instruments. SMEI brightness analyses provided by shortterm image subtractions ("difference images") and, alternatively, subtractions of a mean-brightness fit over a long-time duration, both show the extents of the CMEs travelling outward above the East limb that erupted from the Sun on 24 and 25 January 2007. The SMEI 3-D-reconstructions not only enhance distinct features within the CME events, but also reconcile difference-imaging results with those where a long-term base has been removed. In the January 2007 example the structure as mapped by CME difference images traces the sharp intensity gradients at the front of the CMEs; generally brighter ejected material follows behind the location of the CME front, but shows poorly in these because of its larger angular extent. Using the long-duration background removal enables SMEI's 3-D analysis to determine a mass for this CME sequence North of the ecliptic.
\end{abstract}

Keywords. Interplanetary physics (Flare and stream dynamics; Interplanetary shocks) - Solar physics, astrophysics, and astronomy (Flares and mass ejections)

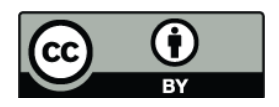

Correspondence to: B. V. Jackson (bvjackson@ucsd.edu)

\section{Introduction}

In this article we use the 24-25 January 2007 coronal mass ejection (CME) event sequence to highlight observations and three-dimensional (3-D) results obtained from the Solar Mass Ejection Imager (SMEI) (Eyles et al., 2003; Jackson et al., 2004). The SMEI instrument is well into its sixth year, recording $0.1 \%$ photometric-quality data covering nearly the entire sky each 102-min orbit. SMEI direct white-light images are available at the University of California, San Diego (UCSD) from early February 2003 to the present on the Website: http://smei.ucsd.edu/. In addition, this Website includes higher-level products from SMEI 3-Dreconstructed volumetric data such as ecliptic and meridional cuts, remote observer views, and Sun-centred fisheye and Hammer-Aitoff projections. The "broad brush" approach to the volumetric analyses provided on the UCSD Website shows both CMEs and corotating structures in 3-D, and provides animations and individual images of CMEs that have been studied by others using image-differencing techniques. Data from SMEI are of particular interest now that the Solar TErrestrial RElations Observatory (STEREO) spacecraft (Kaiser et al., 2008) are launched and returning data. Starting with Carrington rotation (CR) 2054 in April 2007, the Website includes the location of the twin STEREO spacecraft in ecliptic cuts of the higher-resolution reconstruction analyses.

SMEI sky maps are produced from a composite of 4-s exposure $3^{\circ} \times 60^{\circ}$ images from each of the three cameras on board the Coriolis spacecraft (see Fig. 1). The sky maps provide Thomson-scattering data with minimal stray light as close as $20^{\circ}$ elongation to the Sun and as distant from it as $180^{\circ}$. Camera outages occur in SMEI camera 3 (the camera closest to the Sun) when a shutter closes so that the CCD camera is not saturated by sunlight. All cameras fail to obtain data when the moon is in or within a few degrees of their field of view and when a few bright planets saturate the camera response.

Published by Copernicus Publications on behalf of the European Geosciences Union. 
(a)

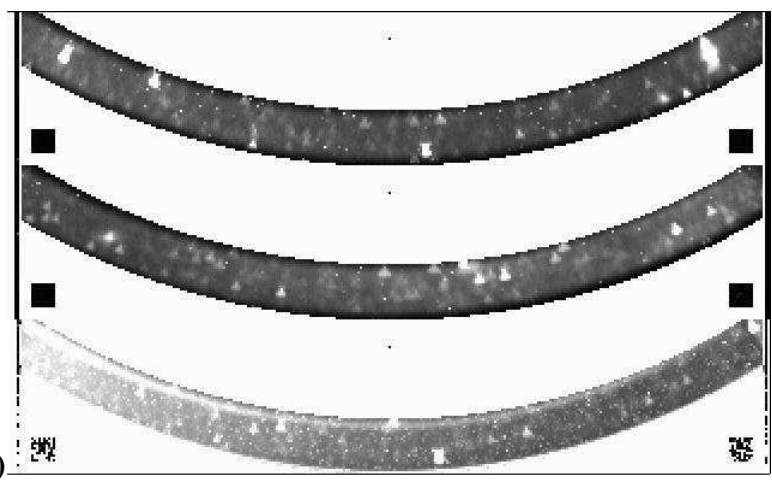

(b)

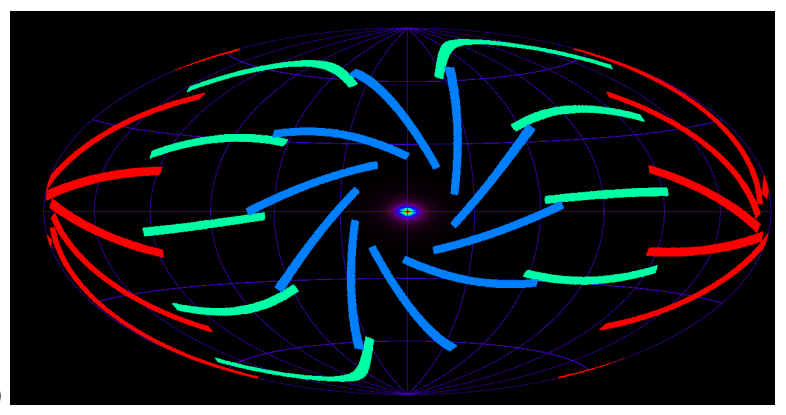

Fig. 1. (a) $3^{\circ} \times 60^{\circ}$ image frames from each of the three SMEI cameras. Camera 1 (top) views farthest from the Sun; and camera 3 (bottom) closest, with the Sun towards the left in each frame. (b) Full-sky Hammer-Aitoff projection showing the placement of a sample of SMEI camera image frames with the Sun centred in the projection (in October). About 4500 frames per orbit fill in the sky in a clockwise direction every $102 \mathrm{~min}$. Camera 1 is shown in red, camera 2 is in green, and camera 3 is in blue.

To reduce long-term brightness variations in SMEI data, a zodiacal-light model has been derived from these data (Hick et al., 2005; Buffington et al., 2009) and removed from them. The data are then fitted at each chosen sidereal location with a long-term Gaussian filter that provides a temporal base with a time constant of several hundred orbits (about 2 weeks). The selected sidereal locations are free of known bright stars, and have high-energy particle hits and auroral light edited and removed from the time series. For more information about this procedure see Jackson et al. (2008).

We have overcome several SMEI data-analysis problems including an unexpectedly hot camera 3. For this, by employing a processing procedure whereby a dark-current pattern updated every orbit is subtracted from the original $3^{\circ} \times 60^{\circ}$ image data, most of the $0.1 \%$ photometric specification can be recovered. This procedure has led to a pronounced enhancement in the quality of SMEI sky maps near the Sun, and this is important in the current context since it allows viewing as near as possible to the Sun, and hence structures that move outward from the solar limbs and even away from the Earth. For the other two cameras, SMEI performs to its original $0.1 \%$ photometric specification over most of the sky (Buffington et al., 2006, 2007, 2009).

Additional data analysis problems have arisen from background noise because of high-energy particle hits at the $840 \mathrm{~km}$ orbital altitude of SMEI, especially when passing through the South Atlantic Anomaly (SAA), and the presence of high-altitude aurora light signals unexpected at this high altitude (Mizuno et al., 2005). The UCSD analysis provides a technique for cleaning high-energy particle hits and aurorae by comparing the redundant images that occur at a given sky location, by recognizing times when high-energy particles hit covered (dark current) CCD pixels, and by orbital timing (see Buffington et al., 2007; Hick et al., 2007; and Jackson et al., 2006, 2008).

The primary focus of the UCSD analyses has been threedimensional (3-D) reconstructions and modelled 2-D images from selected time periods (e.g., Jackson et al., 2004, 2006, 2008; Webb et al., 2009a). However, the SMEI team also produces significant scientific results directly from the 2-D sky map orbit differences (e.g., Tappin et al., 2004; Reiner et al., 2005; Tappin, 2006; Webb et al., 2006, 2009b; Tappin and Howard, 2009).

\section{Data sets used and the comparison of the 24-25 Jan- uary 2007 CME sequence}

We show measurements obtained from SMEI difference images, and the STEREO Sun-Earth Connection Coronal Heliospheric Investigation (SECCHI) instrument suite (Howard et al., 2008). SECCHI provides the means for continuous observations of CMEs from the Sun to Earth. The field of view from the SECCHI Heliospheric Imagers (HIs) (Eyles et al., 2009) is limited in latitude; SMEI has an all-sky imaging capability outward from $20^{\circ}$ elongation. Here as an example, we show a comparison of image differencing techniques for a specific CME event sequence that erupted from the solar East limb on 24 and 25 January 2007. At this time close to the launch of the twin STEREO spacecraft in October 2006, the perspective views from SMEI and STEREO are the approximately the same, and both SMEI and HIA image differencing techniques have been shown to provide approximately the same location and morphology of this CME sequence in the ecliptic plane from both instruments as it moves outward from the Sun (e.g., Webb et al., 2009a). These CMEs were also viewed and measured nearer the solar surface by the Large Angle Spectrographic Coronagraphs (LASCO) (Brueckner et al., 1995) on board the SOlar and Heliospheric Observatory (SOHO) spacecraft (Domingo et al., 1995) nearer the solar surface.

As in many CME-event sequences, because of data outages and incomplete coverage in both the SMEI and HI instrumentation, it is difficult to discern the true extent of the CME sequence and to connect the portions of it in position angle (PA) that progress outward across the sky. In earlier 

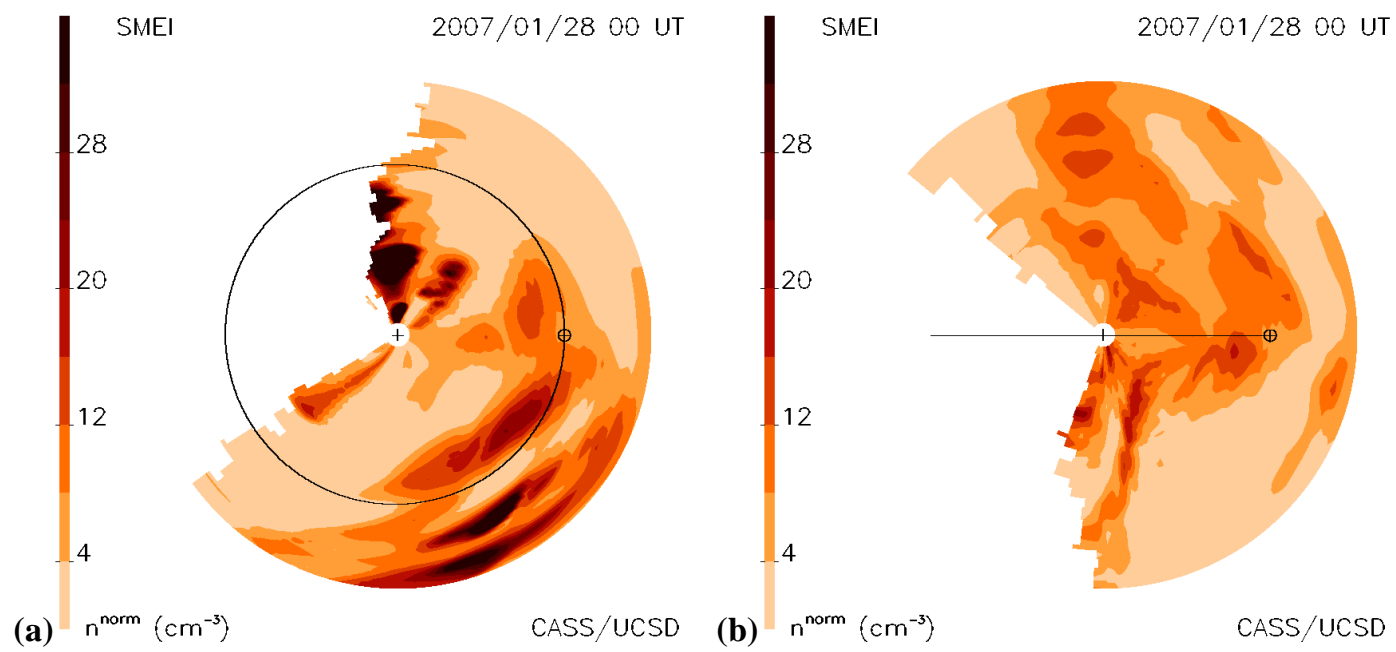

Fig. 2. Ecliptic and meridional cuts of the 24-25 January $2007 \mathrm{CME}$ at the time indicated when the main portions of the CMEs have coalesced in the ecliptic plane and have reached 1 AU East of the Sun. The Earth is marked " $\otimes$ " and the Sun a "+" in the centre of each plot. Densities are given in protons (in $\mathrm{cm}^{-3}$ ) and are normalised to the measurements at 1 AU taking an $r^{-2}$ fall-off in the density into account. The areas to the left in each image where fewer than 10 lines of sight cross are left blank as on the UCSD Website at http://smei.ucsd.edu/. (a) Ecliptic cut showing the Earth orbit as an ellipse. (b) Meridional cut showing the Earth and its orbit as a horizontal line terminating at the Earth.

studies of the 24-25 January 2007 CME event sequence (e.g., Webb et al., 2009a) only piecemeal portions of the events are shown as they move outward from the Sun and/or the elongation of the events mapped on direct images do not match well with measurements of them where a long duration base has been subtracted from the brightness data in UCSD 3-D reconstructions. In this paper for the first time we provide difference images of this event sequence from the UCSD 3$\mathrm{D}$ reconstructions in order to provide measurements of the fronts of the CMEs as determined using direct image differencing techniques. We also show how the SMEI measurements of 3-D CME mass obtained by removing a long-term temporal base from the brightness data compare with values of mass obtained for this CME event sequence using LASCO C3 coronagraph observations.

\section{The UCSD 3-D-reconstruction technique}

The UCSD 3-D-reconstruction technique with our standard kinematic model has been used to determine global 3-D densities and velocities in the inner heliosphere using a timedependent model since 1999 (Jackson et al., 2001). Given the velocity and density of an inner boundary (the "source surface"), a 3-D-solar-wind model best fitting the observations is calculated, assuming radial outflow and enforcing conservation of mass and mass flux (Jackson et al., 1998). Best fit is achieved iteratively. If the line-of-sight integrations of 3-D solar wind at large solar distances do not match the overall observations, the source-surface values are modified and the deviations reduced to a desired small value. Extensive study of this process has shown that the final iterated values are insensitive to the starting values on the source surface.

This technique has been used to analyse CME-associated structures using interplanetary scintillation (IPS) and SMEI Thomson-scattering observations (e.g., Jackson and Hick, 2005). Different Gaussian filters are used for the two data sets of SMEI and IPS that limit the size of structures observed to a larger volumetric shape than the digital resolution (see Jackson and Hick, 2005; Jackson et al., 2006).

In addition to the SMEI-observed time-varying signal, the reconstruction includes an unchanging background component. This is estimated by relating model densities to in-situ measurements near Earth. An average in-situ measurement of the solar wind density determines the average background brightness while the variable in-situ component is related to the heliospheric brightness change. SMEI has now been calibrated (Buffington et al., 2007) to $4 \%$ absolute for all three of its cameras. The heliospheric model produced by SMEI matches Advanced Composition Explorer (ACE) (Stone et al., 1998) and Wind (Ogilvie and Desch, 1997) proton density data well (e.g., Jackson et al., 2007; 2008; Bisi et al., 2008). To match spacecraft proton density, the UCSD model comparisons include not only the calibration of the SMEI brightness observations, but also an assumption that the heliosphere has a $10 \%$ Helium abundance thus providing $20 \%$ more electrons in the heliosphere from proton abundance alone. Successful comparison of 3-D-reconstructions and insitu measurements to within $\sim 5 \%$ of the proton number density $\left(N_{P}\right)$ for the 28 May 2003 CME (Jackson et al., 2008) and 4-8 November 2004 CME event sequences (Bisi et al., 2008) confirms that SMEI brightness is calibrated correctly 


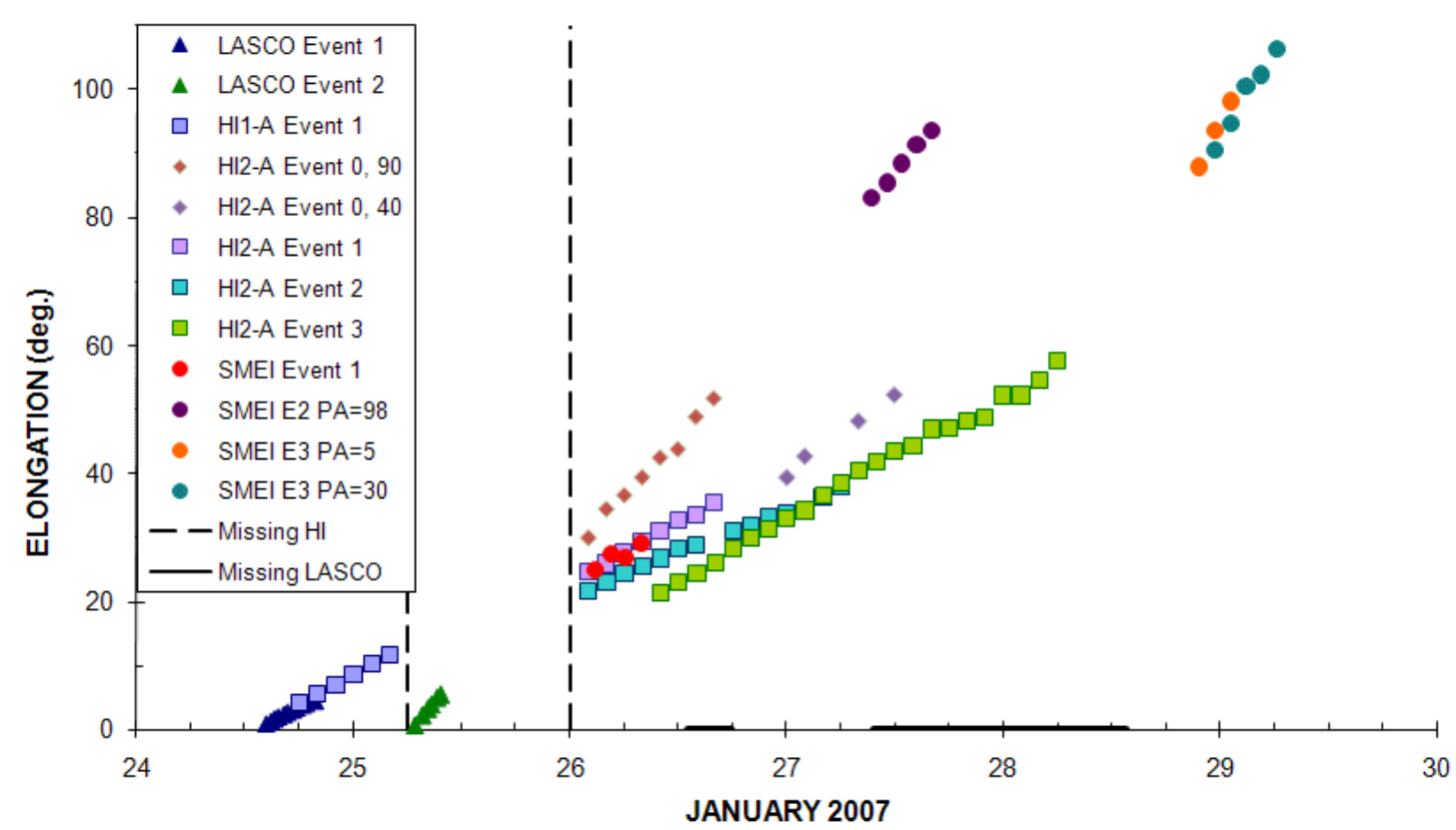

Fig. 3. Elongation-time plot from Webb et al. (2009a) showing a variety of structures in late-January 2007, measured by image-differencing techniques using STEREO HI-1A and HI-2A and SMEI observations for the CMEs mapped from near the surface of the Sun to beyond $90^{\circ}$ elongation. Some portions of the same CME structure overlap and can be measured in both HI-1A and SMEI. Pertinent here are the structures SMEI E2 PA $=98^{\circ}$, SMEI E3 PA $=5^{\circ}$, and SMEI E3 PA $=30^{\circ}$, which were effectively measured using SMEI imaging-difference techniques. In the 3-D-reconstruction analyses these are shown to be part of the same large loop structure that has not advanced to as large an elongation to the solar North as it has to the solar East.

and that SMEI obtains the proper density using these 3-D reconstructions. The SMEI website currently provides the higher-level 3-D data analyses using this conversion from electron to proton densities in order to better match the insitu density values measured by spacecraft.

\section{Comparison of remote-sensing images and 3-D- reconstructions: SMEI and STEREO}

Difference images from SMEI and from the STEREO HI-2A instrument (Howard et al., 2008; Eyles et al., 2009) generally look different and show different structures from those in SMEI where a long-term temporal base averaged from weeks of data has been subtracted. For SMEI, the long-term temporal base subtraction requires strict maintenance of straylight levels and careful removal of background sources of light such as the zodiacal light and stars. This allows the changing CME brightness above a mean level to be determined. Thus these brightness measurements can be directly related to bulk density above the ambient solar wind density that itself can be accurately modelled by comparison with insitu measurements (Jackson et al., 2001, 2008; Jackson and Hick, 2005). This allows display of the volumes in terms of bulk density, and these are shown on the UCSD Website as ecliptic or meridional cuts of the volumes as shown in Fig. 2 for the 24-25 January 2007 CME event sequence.
In a recent article Webb et al. (2009a) show elongationtime plots of the 24 and 25 January CME sequence (Fig. 3), and trace these observations in the SMEI and STEREO inner Heliospheric Imager (HI-1) and HI-2 difference image data. These show structures in intermittent direct different images from the STEREO HIs and SMEI to the West and North that reach $90^{\circ}$ elongation and beyond. For details of how the SMEI difference imaging is accomplished, used in these earlier analyses, and in further applications see Kuchar et al. (2008). The long-term analyses from the SMEI data cleaned of auroral light and high-energy particle hits and fit using the UCSD solar wind 3-D-reconstruction analysis reveals these structures to be large loops, consistent with their shapes indicated by the various modelling techniques described in Webb et al. (2009a).

The difference between using an image with a long-term base subtracted and one where difference images are used is demonstrated in Fig. 4. Figure 4 shows 3-D-reconstructed fisheye images of the sequence of CMEs during late January 2007. To the North, these loop events are well-defined, but to the South they overlap and coalesce where their images are further complicated by light from the dust and plasma of Comet McNaught (C/2006 P1). By differencing 3-Dreconstructed volumes $12 \mathrm{~h}$ apart, we show in Fig. $4 \mathrm{~b}$ and $\mathrm{d}$ that the fronts of the CMEs are enhanced, and that the locations of these fronts are more-closely related to SMEI 

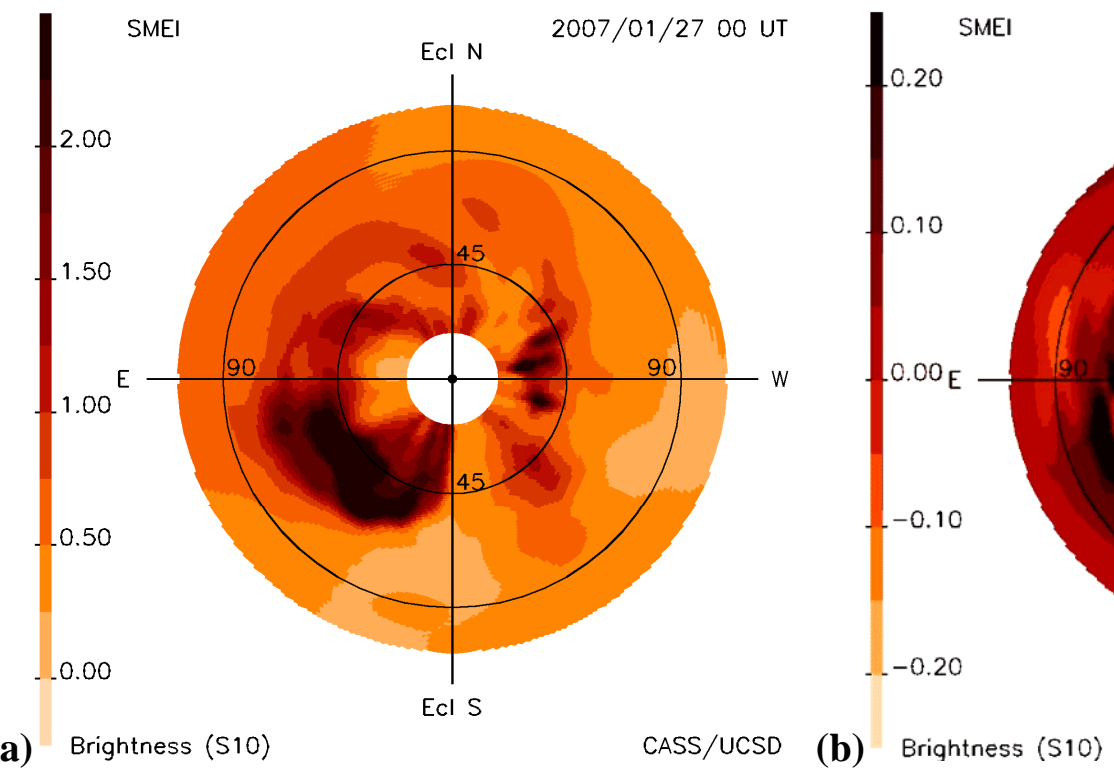

(a)

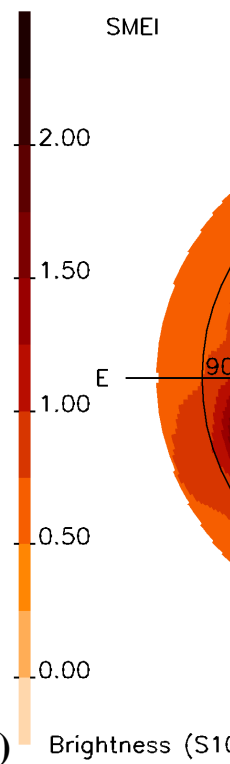

$2007 / 01 / 2812$ UT

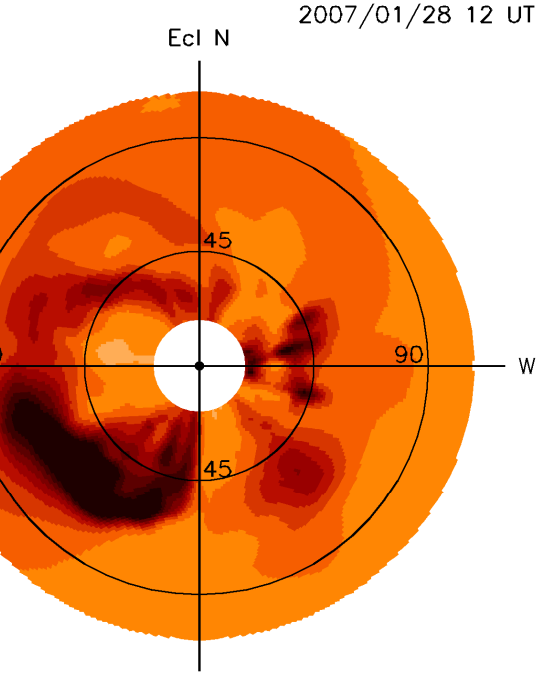

Ecl S

CASS/UCSD

(b)

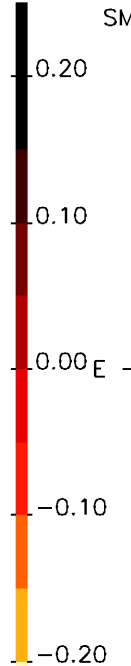

(d)
SME

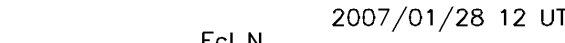

CASS/UCSD

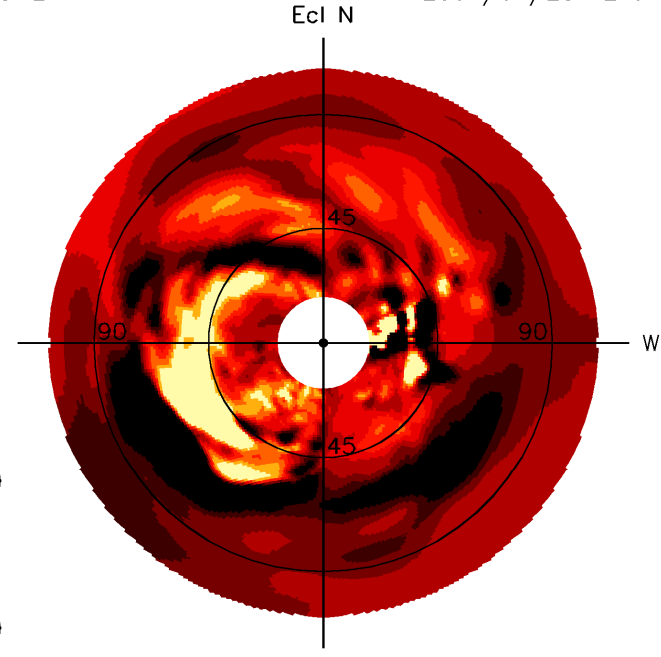

Ecl S

CASS/UCSD

Fig. 4. 3-D-reconstructed Sun-centred fisheye sky maps to $110^{\circ}$ elongation showing the outward progression of several CMEs during $27-28$ January 2007. Brightness in S10 units (tenth magnitude solar-type stars in a square degree of sky) is shown to the left of each image. An $r^{-2}$ density fall-off has been removed here to show the structures. (a) and (c) 3-D image reconstruction with a long-term base removed. The outer-loop composite that reaches to $\sim 60^{\circ}$ elongation East of the Sun in (a) is associated with CMEs on 24 and 25 January 2007 (Webb et al., 2009a). (b) and (d) Fisheye "difference images" as in (a) and (c) obtained after subtracting the 12-h-preceding density volumes from the current volumes. Difference images tend to emphasize the smaller-scale structures. These sky maps highlight the loop fronts of (a) and (c). In particular, the loop front at $90^{\circ}$ elongation to the North from $5^{\circ}$ to $30^{\circ} \mathrm{PA}$ in (d) is at the approximate elongation of the structure measured at these same PAs in SMEI image differences with a shorter-term base removed.

orbit-to-orbit difference images. The generally brighter ejected material of the CMEs follows behind the location of the CME fronts.

\section{CME mass}

With these events now reconstructed fully in three dimensions, we are also able to determine their interplanetary mass (see Fig. 5) as in Jackson et al. (2006, 2008). These measurements provide far more precise 3-D masses for individual 


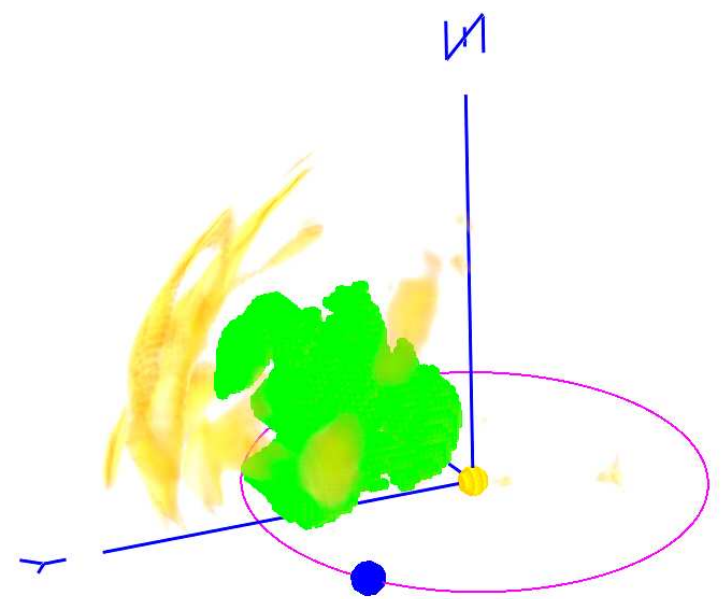

2007/01/27 12:00 UT

$\begin{array}{ll}\text { Excess Mass } & =2.12^{16} \mathrm{~g} \\ \text { Total Mass } & =2.80^{16} \mathrm{~g} \\ \text { Ambient } & =7.02{ }^{15} \mathrm{~g} \\ \text { Volume } & =0.104 \mathrm{AU}^{3}\end{array}$

Fig. 5. Estimates of 3-D mass for the composite CME structure to the north of the ecliptic for the 24-25 January 2007 CME sequence on 27 January 2007 at 12:00 UT. Earth is shown as the blue dot, on its orbit mapped as a purple ellipse. The volumetric material within the $15 N_{p} \mathrm{~cm}^{-3}$ contour interval is approximated by cubes and summed (shown in green) to obtain the result. The values of excess, total, and ambient mass, and the volume highlighted are shown in the figure caption. Portions of pre-existing heliospheric material not connected with the CME structure appear in yellow to the Northeast. Other non-associated material to the South and West of the Sun-Earth line has been removed for clarity of viewing.

events, and also a consistency check to ensure that the CME material observed by SMEI agrees with measurements from other instruments. For the 24 and 25 January 2007 event sequence, the SMEI 3-D analysis measures several CMEs propagating outward that are difficult to separate in 2-D images or 3-D volumes. To the North of the ecliptic, the total mass is a composite of CME material within a $15 N_{P} \mathrm{~cm}^{-3}$ contour level and is $2.8 \times 10^{16} \mathrm{~g}$. We have chosen this contour level such that the composite CME to the North is separated from other structure. Differentiation of this northern structure into individual event components is possible, but only by selecting a higher contour level to further isolate event features. If this is done, the material below the higher contour level does not include intervening (supposedly merged) material from the separate CMEs. The volume enclosed by this event sequence to the North within the $15 N_{P} \mathrm{~cm}^{-3}$ contour level is $\sim 0.10 \mathrm{AU}^{3}$ at 12:00 UT 27 January 2007 . Within this contour level the modelled ambient mass $\left(7.0 \times 10^{15} \mathrm{~g}\right)$ of the solar wind comprises about $25 \%$ of the total CME mass. No attempt to estimate mass to the South of the ecliptic plane was made because of the uncertainty in the background contribution of dust from comet McNaught.
The excess mass to the North of $2.1 \times 10^{16} \mathrm{~g}$ (see Fig. 5) observed by SMEI compares favourably with the excess mass derived from the LASCO C 3 coronagraph on board the SOHO spacecraft. The plane of the sky mass derived from LASCO C3 coronagraph as given in Webb et al. (2009a) for the two events totals $2.0 \times 10^{16} \mathrm{~g}$ excess mass. Since the SMEI analysis reconstructs considerable mass for these events that is distant from the coronagraph sky plane, this sky-plane mass estimate must be adjusted considerably (e.g., see Jackson et al., 2008), and is most likely to increase by a factor of at least 1.5. Thus, the northern portion of this CME excess mass measured in SMEI is certainly consistent with its being a portion of the excess mass observed in LASCO. Lugaz et al. (2008) argue that this same event sequence is additionally influenced in the ecliptic by corotating structures that contribute to the brightness differences observed in the $\mathrm{HI}-2 \mathrm{~A}$ instrument. Indeed a portion of the mass observed in the SMEI analyses on 27 January in Fig. 5 could be attributed to corotating solar wind features, However, we note that very little of the bulk of mass is observed to corotate in our ecliptic-cut analysis of this event even though the 3-Dreconstruction analysis can (and often does) show corotation in these ecliptic cuts.

These SMEI analyses can be used to estimate a kinetic energy for the CME outward flow. Because no IPS velocity data are available for the time period covered by these reconstructions, as in the case of the events measured by Jackson et al. (2006, 2008), kinetic energy for this event is estimated by assuming a constant velocity for the whole of the CME structure. We generally assume a velocity of the material near $1 \mathrm{AU}$ of $400 \mathrm{~km} \mathrm{~s}^{-1}$ (the ambient mean for solar wind velocity structure). This velocity implies a $1 / 2 \mathrm{mv}^{2}$ kinetic energy of $2.2 \times 10^{31} \mathrm{erg}$ associated with the total mass outward flow for the northern part of this event sequence. Since no attempt was made to determine CME mass to the South of the ecliptic plane for this event because of the uncertain dust contribution from comet McNaught, we likewise do not estimate the energy associated with this portion of the CME.

\section{Conclusions}

Many of these SMEI analyses shown in this paper can be found on the UCSD Website at: http://smei.ucsd.edu/ for the entire six plus years of SMEI data. These include results from 3-D-reconstructed volumetric data such as ecliptic and meridional cuts, remote-observer views, and Suncentred fisheye and Hammer-Aitoff projections. In this paper we have presented observations and analyses of SMEI, STEREO and LASCO data from the 24-25 January 2007 CME sequence. During this period, the STEREO spacecraft views are aligned approximately with those from SMEI. Using difference images, several CME structures that have been mapped outward in Webb et al. (2009a) coincide in both the HI-2A and SMEI observations, but in this analysis 
the elongation-time plots of Fig. 3 show structures that are mapped primarily as differently-directed separate entities. In the SMEI 3-D reconstructions shown in Sect. 4, structure locations are determined either by short-term 12-h subtractions, or by subtracting a mean brightness fit over a long-time duration. SMEI 3-D reconstructions show the CMEs as giant loop-like structures that extend from the North of the Sun and far to the South. This is consistent with model results from Lugaz et al. (2008), HAF-model estimates (Hakamada and Akasofu, 1982; Fry et al., 2001, 2003), and ENLILmodel simulations (e.g., Odstrcil et al., 2003) shown in Webb et al. (2009a), all of which show a similar loop-like structure for the CME response. We show in this case that the structures mapped by difference images primarily follow the sharp intensity gradients at the front of the CMEs, something not evident in the reconstructed images that are not differenced. This agrees with the short-term difference images obtained from SMEI towards the solar North used to provide elongation-time plots in Webb et al. (2009a). Since we have measurements relative to a long term base from our calibrated SMEI analysis we are able to determine estimates of the 3-D total and excess mass for this CME sequence in the North heliospheric hemisphere. With the caveat that we have defined the volumetric material for the CMEs to exist within a $15 N_{P} \mathrm{~cm}^{-3}$ contour interval, we find values of $2.1 \times 10^{16} \mathrm{~g}$ and $2.8 \times 10^{16} \mathrm{~g}$ for excess and total CME mass respectively for this CME event sequence to the North. The excess mass determined for the CME sequence agrees roughly with that measured using LASCO C3 difference images for this event. The total CME mass and an estimate of the outward motion of the CME give a kinetic energy associated with the CME sequence to the North of $2.2 \times 10^{31} \mathrm{erg}$.

Acknowledgements. We thank D. F. Webb of Boston College, USA for a careful reading of the initial manuscript, and the SMEI team for providing continuing data acquisition from the SMEI instrument. We also thank C. Eyles of the University of Birmingham, UK, for SECCHI HI data, J. Davies of the Rutherford Appleton Laboratory (RAL), UK for HI image processing, and V. Kunkel of the Naval Research Laboratory (NRL), Washington D.C., USA for SECCHI distance - time measurements used in initial comparisons. SMEI was designed and constructed by a team of scientists and engineers from the US Air Force Research Laboratory, the University of California at San Diego, Boston College, Boston University, and the University of Birmingham, UK. The STEREO/SECCHI project is an international consortium of NRL (USA), Lockheed Martin Solar and Astrophysics Lab (USA), NASA Goddard Space Flight Center (USA), RAL (UK), University of Birmingham (UK), Max-Planck-Institut für Sonnensystemforschung (Germany), Centre Spatial de Liège (CSL) (Belgium), Institut d'Optique Théorique et Appliquée (France), and Institut d'Astrophysique Spatiale (France). The SECCHI HI instruments were developed by a collaboration that included the University of Birmingham, RAL, CSL, and NRL. The work of B. V. Jackson, A. Buffington, P. P. Hick, and J. M. Clover was supported at the University of California, San Diego by NSF grant ATM-0331513 and NASA grants NAG5-134543 and NNG05GG45G. Post-doctoral fellow M.M. Bisi was supported in part by AFOSR grant FA955006-1-0107 and NASA grant NNG05GG45G.

Topical Editor R. Forsyth thanks N. Srivastava and another anonymous referee for their help in evaluating this paper.

\section{References}

Bisi, M. M., Jackson, B. V., Hick, P. P., Buffington, A., Odstrcil, D., and Clover, J. M.: 3D Reconstructions of the Early-November 2004 CDAW Geomagnetic Storms: Analyses of STELab IPS Speed and SMEI Density Data, J. Geophys. Res., 113, A00A11, doi:10.1029/2008JA013222, 2008.

Brueckner, G. E., Howard, R. A., Koomen, M. J., Korendyke, C. M., Michels, D. J., Moses, J. D., Socker, D. G., Dere, K. P., Lamy, P. L., Llebaria, A., Bout, M. V., Schwenn, R., Simnett, G. M., Bedford, D. K., and Eyles, C. J., The Large Angle Spectroscopic Coronagraph (LASCO): visible light coronal imaging and spectroscopy, Solar Phys., 162, 357-402, 1995.

Buffington, A., Band, D. L., Jackson, B. V., Hick, P. P., and Smith, A. C.: A Search for Early Optical Emission at Gamma-Ray Burst Locations by the Solar Mass Ejection Imager (SMEI), Astrophys. J., 637, 880, doi:10.1086/498407, 2006.

Buffington, A., Morrill, J. S., Hick, P. P., Howard, R. A., Jackson B. V., and Webb, D. F.: Analysis of the Comparative Responses of SMEI and LASCO, Proc. SPIE 6689, 66890B, 1, doi:10.1117/12.734658, 2007.

Buffington, A., Bisi, M. M., Clover, J. M., Hick, P. P., Jackson, B. V., Kuchar, T. A., and Price, S. D.: Measurements of the Gegenschein brightness from the Solar Mass Ejection Imager (SMEI), Icarus, 203, 124, doi:10.1016/j.icarus.2009.04.007, 2009.

Domingo, V., Fleck, B., and Poland, A. I.: SOHO: The Solar and Heliospheric Observatory, Space Sci. Rev., 72, 81-84, 1995.

Eyles, C. J., Simnett, G. M., Cooke, M. P., Jackson, B. V., Buffington, A., Hick, P. P., Waltham, N. R., King, J. M., Anderson, P. A., and Holladay, P. E., The Solar Mass Ejection Imager (SMEI), Solar Phys., 217, 319, doi:10.1023/B:SOLA.0000006903.75671.49, 2003.

Eyles, C. J., Harrison, R. A., Davis, C. J., Waltham, N. R., Shaughnessy, B. M., Mapson-Menard, H. C. A., Bewsher, D., Crothers, S. R., Davies, J. A., Simnett, G. M., Howard, R. A., Moses, J. D., Newmark, J. S., Socker, D. G., Halain, J. P., Defise, J. M., Mazy, E., and Rochus, P.: The Heliospheric Imagers On-board the STEREO Mission, Solar Phys., 254, 387, doi:10.1007/s11207008-9299-0,, 2009.

Fry, C. D., Sun, W., Deehr, C. S., Dryer, M., Smith, Z., Akasofu, S.-I., Tokumaru, M., and Kojima, M.: Improvements to the HAF Solar Wind Model for Space Weather Predictions, J. Geophys. Res., 106, 20985-21001, 2001.

Fry, C. D., Dryer, M., Smith, Z., Sun, W., Deehr, C. S., and Akasofu, S.-I.: Forecasting Solar Wind Structures and Shock Arrival Times Using an Ensemble of Models, J. Geophys. Res., 108(A2), 1070, doi:10.1029/2002JA009474, 2003.

Hakamada, K. and Akasofu, S.-I.: Simulation of ThreeDimensional Solar Wind Disturbances and Resulting Geomagnetic Storms, Space Sci. Rev., 31, 3-70, 1982.

Hick, P. P., Buffington, A., and Jackson, B. V.: The SMEI real-time data pipeline: From raw CCD frames to photometrically accurate full-sky maps, Proc. SPIE, 59011B, doi:10.1117/12.617996, 2005. 
Hick, P. P., Buffington, A., and Jackson, B. V.: A Procedure for Fitting Point Sources in SMEI White-Light Full-Sky Maps, Proc. SPIE 6689, 66890C, 1, 2007.

Howard, R. A., Moses, J. D., Vourlidas, A., Newmark, J. S., Socker, D. G., Plunkett, S. P., et al.: Sun Earth Connection Coronal and Heliospheric Investigation (SECCHI), Space Sci. Rev., 136, 67$115,2008$.

Jackson, B. V. and Hick, P. P.: Three-dimensional tomography of interplanetary disturbances, Chapter 17, in: Solar and Space Weather Radiophysics, Current Status and Future Developments, Astrophysics and Space Science Library, edited by: Gary, D. E. and Keller, C. U., 314, 355-386, Kluwer Academic Publ., Dordrecht, The Netherlands, 2005.

Jackson, B. V., Hick, P. L., Kojima, M., and Yokobe, A.: Heliospheric Tomography Using Interplanetary Scintillation Observations 1. Combined Nagoya and Cambridge data, J. Geophys. Res., 103, 12049-12067, 1998.

Jackson, B. V., Buffington, A., and Hick, P. P.: A heliospheric imager for Solar Orbiter, Proc. "Solar Encounter: The First Solar Orbiter Workshop", May 14-18, 2001, Santa Cruz de Tenerife, Spain, ESA SP-493, 251, 2001.

Jackson, B. V., Buffington, A., Hick, P. P., Altrock, R. C., Figueroa, S., Holladay, P. E., Johnston, J. C., Kahler, S. W., Mozer, J. B., Price, S., Radick, R. R., Sagalyn, R., Sinclair, D., Simnett, G. M., Eyles, C. J., Cooke, M. P., Tappin, S. J., Kuchar, T., Mizuno, D., Webb, D. F., Anderson, P. A., Keil, S. L., Gold, R. E., and Waltham, N. R.: The Solar Mass Ejection Imager (SMEI) Mission, Solar Phys., 225, 177, doi:10.1007/s11207-004-2766-3, 2004

Jackson, B. V., Buffington, A., Hick, P. P., Wang, X., and Webb, D.: Preliminary three-dimensional analysis of the heliospheric response to the 28 October 2003 CME using SMEI white-light observations, J. Geophys. Res., 111, A04S91, doi:10.1029/2004JA010942, 2006.

Jackson, B. V., Buffington, A., Hick, P. P., Bisi, M. M., and Jensen, E. A.: SMEI Observations in the STEREO Era, in Proc. SPIE 6689, 66890C, 1, 2007.

Jackson, B. V., Bisi, M. M., Hick, P. P., Buffington, A., Clover, J. M., and Sun, W.: Solar Mass Ejection Imager (SMEI) 3D reconstruction of the 27-28 May 2003 CME sequence, J. Geophys Res., 113, A00A15, doi:10.1029/2008JA013224, 2008.

Kaiser, M. L., Kucera, T. A., Davila, J. M., St. Cyr, O. C., Guhathakurta, M., and Christian, E.: The STEREO Mission: An Introduction, Space Sci. Rev., 136, 5-16, 2008.

Kuchar, T. A., Buffington, A., Arge, C. N., Hick, P. P., Howard, T. A., Jackson, B. V., Johnston, J. C., Mizuno, D. R., Tappin, S. J., and Webb, D. F.: Observations of a Comet Tail Disruption Induced by the Passage of a CME, J. Geophys. Res., 113, A04101, doi:10.1029/2007JA012603, 2008.

Lugaz, N., Vourlidas, A., Roussev, I. I., Jacobs, C., Manchester IV, W. B., and Cohen, O.: The Brightness of Density Structures at Large Solar Elongation Angles: What Is Being Observed by STEREO SECCHI?, Astrophys. J., 684, L111, doi:10.1086/592217, 2008.
Mizuno, D. R., Buffington, A., Cooke, M. P., Eyles, C. J., Hick, P. P., Holladay, P. E., Jackson, B. V., Johnston, J. C., Kuchar, T. A., Mozer, J. M., Price, S. D., Radick, R. R., Simnett, G. M., Sinclair, D., and Webb, D. F.: Very High-Altitude Aurora Observations With The Solar Mass Ejection Imager, J. Geophys. Res., 110, A07230, doi:10.1029/2004JA010689, 2005.

Odstrcil, D., Riley, P., Linker, J. A., Lionello, R., Mikic, Z., and Pizzo, V. J.: 3-D simulations of ICMEs by coupled coronal and heliospheric models, in: Solar Variability as an Input to the Earth's Environment, edited by: Wilson, A., Estec, The Netherlands, SP-535, ESA, Noordwijk, 541, 2003.

Ogilvie, K. W. and Desch, M. D.; The Wind Spacecraft and its Early Scientific Results, Adv. Space Res., 20(445), 559-568, 1997.

Reiner, M. J., Jackson, B. V., Webb, D. F., Mizuno, D. R., Kaiser, M. L., and Bougeret, J. L.: CME Kinematics Deduced from White-Light (SMEI) and Radio (Wind/WAVES) Observations, J. Geophys. Res., 110(A9), A09S14, doi:10.1029/2004JA010943, 2005.

Stone, E. C., Frandsen, A. M., Mewaldt, R. A., Christian, E. R., Margolies, D., Ormes, J. F., and Snow, F.: The Advanced Composition Explorer, Space Sci. Rev., 86, 1-22, 1998.

Tappin, S. J.: The Deceleration of an Interplanetary Transient from the Sun to 5 AU, Solar Phys., 233, 233, doi:10.1007/s11207-0062065-2, 2006.

Tappin, S. J. and Howard, T. A.: Interplanetary Coronal Mass Ejections Observed in the Heliosphere: 2. Model and Data Comparison, Space Sci. Rev., in press, doi:10.1007/s11214-009-9550-5, 2009.

Tappin, S. J., Buffington, A., Cooke, M. P., Eyles, C. J., Hick, P. P. Holladay, P. E., Jackson, B. V., Johnston, J. C., Kuchar, T., Mizuno, D., Mozer, J. B., Price, S., Radick, R. R., Simnett, G. M., Sinclair, D., Waltham, N. R., and Webb, D. F.: Tracking a Major Interplanetary Disturbance with SMEI, Geophys. Res. Lett., 31, L2802, doi:10.1029/2003GL018766, 2004.

Webb, D. F., Mizuno, D. R., Buffington, A., Cooke, M. P. Eyles, C. J., Fry, C. D., Gentile, L. C., Hick, P. P., Holladay, P. E., Howard, T. A., Hewitt, J. G., Jackson, B. V., Johnston, J. C., Kuchar, T. A., Mozer, J. B., Price, S., Radick, R. R. Simnett, G. M., and Tappin, S. J.: Solar Mass Ejection Imager (SMEI) Observations of CMEs in the Heliosphere, J. Geophys. Res., 111, A12101, doi:10.1029/2006JA011655, 2006.

Webb, D. F., Howard, T. A., Fry, C. D., Kuchar, T. A., Odstrcil, D., Jackson, B. V., Bisi, M. M., Harrison, R. A., Morrill, J. S., Howard, R. A., and Johnston, J. C.: Study of CME Propagation in the Inner Heliosphere: SMEI and STEREO HI Observations of the January 2007 Events, Solar Physics, STEREO Special Issue, 256, 239, doi:10.1007/s11207-009-9351-8, 2009a.

Webb, D. F., Howard, T. A., Fry, C. D., Kuchar, T. A., Mizuno, D. R., Johnston, J. C., and Jackson, B. V.: Studying Geoeffective ICMEs between the Sun and Earth: Space Weather Implications of SMEI Observations, Space Weather, 7, S05002, doi:10.1029/2008SW000409, 2009b. 